\title{
Almost Unbiased Ratio cum Product Estimator for Finite Population Mean with Known Median in Simple Random Sampling
}

\author{
Jambulingam Subramani ${ }^{1}$ and Master Ajith $\mathbf{S}^{2^{*}}$
}

Submitted: 4 May 2017; Accepted: 23 July 2017

\begin{abstract}
Introduction: In sampling theory, different procedures are used to obtain the efficient estimator of the population mean. The commonly used method is to obtain the estimator of the population mean is simple random sampling without replacement when there is no auxiliary variable is available. There are methods that use auxiliary information of the study characteristics. If the auxiliary variable is correlated with study variable, number of estimators are widely available in the literature.

Objective: This study deals with a new ratio cum product estimator is developed for the estimation of population mean of the study variable with the known median of the auxiliary variable in simple random sampling.

Materials and Methods: The bias and mean squared error of proposed estimator are derived and compared with that of the existing estimators by analytically and numerically.

Results: The proposed estimator is less biased and mean squared error is less than that of the existing estimators and from the numerical study, under some known natural populations, the bias of proposed estimator is approximately zero and the mean squared error ranged from 6.83 to 66429.21 and percentage relative efficiencies ranged from 103.65 to 2858.75 .

Conclusion: The proposed estimator under optimum conditions is almost unbiased and performs better than all other existing estimators.
\end{abstract}

Keywords: Bias, mean squared error, natural populations, product estimator, ratio estimator, simple random sampling.

Address correspondence to the authors: Department of Statistics, Ramanujan School of Mathematical Sciences, Pondicherry University, Puducherry, 605014, India.

Email: drjsubramani@yahoo.co.in ${ }^{1}$; ajith.master9@gmail.com ${ }^{2 *}$ (corresponding author email) 


\section{INTRODUCTION}

The main aim of sampling theory is to obtain precise results about the parameters of study variable on the basis of random samples. One can use the parameters of auxiliary variable such as mean, median, variance, the coefficient of variation, correlation coefficient etc. are used to improve the efficiency of the estimators of study variable. Let in a finite population having $\mathrm{N}$ units $\left\{U_{1}, U_{2}, U_{3} \ldots U_{N}\right\}$. Y be the study variable and $\mathrm{X}$ be the auxiliary variable which is correlated with $\mathrm{Y}$. If $y=\left\{y_{1}, y_{2}, y_{3} \ldots y_{n}\right\}$, and $x=\left\{x_{1}, x_{2}, x_{3} \ldots x_{n}\right\}$ be $\mathrm{n}$ sample values. When the population mean of the auxiliary variable is known, different estimators such as ratio, product and linear regression estimators are proposed in the literature. Among all these estimators the ratio estimator and its modifications are widely used for the estimation of the mean of the study variable. This work is focused on improving the efficiency of some ratio cum product estimators in the literature by obtaining the optimal value. Cochran (1940), Singh, Tailor and Kakran (2004), Kadilar and Cingi (2004, 2006), Subramani (2013), Subramani and Kumarapandiyan (2012a, 2012b), Subramani and Master Ajith (2016a, 2016b), Upadhyaya and Singh (1999), Yan and Tian (2010), Sisodia and Dwivedi (1981), etc. suggested different class of estimators of the population mean with known parameters of the auxiliary variable.

Before discussing further the various estimators, the notations to be used in this article are listed here.

\begin{tabular}{|c|c|c|c|c|c|}
\hline $\mathrm{N}$ & - & Population size & $\beta_{2}$ & - & Coefficient of kurtosis \\
\hline $\mathrm{n}$ & - & Sample size & $M$ & - & Median of study variable \\
\hline$f=\frac{n}{N}$ & - & Sampling fraction & $M_{d}$ & - & Median of auxiliary variable \\
\hline Y & - & Study variable & $\hat{\bar{Y}}_{P r}$ & - & Proposed estimator \\
\hline $\mathrm{X}$ & - & Auxiliary variable & $\bar{y}_{s r s}$ & - & Simple random sampling \\
\hline $\bar{X}, \bar{Y}$ & - & Population means & & & (WOR) estimator \\
\hline $\bar{x}, \bar{y}$ & - & Sample means & $\hat{\bar{Y}}_{R}$ & - & Ratio estimator \\
\hline$S_{x}, S_{y}$ & - & Population standard deviations & $\hat{\bar{Y}}_{P}$ & - & Proposed estimator \\
\hline$s_{x}, s_{y}$ & - & Sample standard deviations & $\hat{\bar{Y}}_{M R}$ & - & Modified ratio estimator \\
\hline$C_{x}, C_{y}$ & - & Coefficient of variations & $\hat{\bar{Y}}_{M P}$ & - & Modified product estimator \\
\hline \multirow[t]{2}{*}{$\rho$} & - & Correlation coefficient between & $\mathrm{B}()$. & - & Bias of estimators \\
\hline & & $\mathrm{x}$ and $\mathrm{y}$ & MSE(.) & - & Mean squared error of \\
\hline$\beta_{1}$ & - & Coefficient of skewness & & & estimators \\
\hline
\end{tabular}


The simple random sample mean without replacement is used only when there is no additional information of the study variable is available. In simple random sampling the estimator $\bar{y}_{\text {srs }}$ is an unbiased estimator for the population mean $\bar{Y}$ and its variance is

$$
\mathrm{V}\left(\bar{y}_{s r s}\right)=\left(\frac{1-\mathrm{f}}{\mathrm{n}}\right) S_{y}{ }^{2}=\left(\frac{1-\mathrm{f}}{\mathrm{n}}\right) \bar{Y}^{2} C_{y}^{2}
$$

Where $\bar{Y}=\frac{1}{N} \sum_{i=1}^{N} Y_{i}, S_{y}{ }^{2}=\frac{1}{N-1} \sum_{i=1}^{N-1}\left(Y_{i}-\bar{Y}\right)^{2}, C_{y}=\frac{S_{y}}{\bar{Y}}$,

The auxiliary variable and study variable are positively correlated, ratio estimator and their modifications are used. The ratio estimator (Cochran, 1940) is given

$$
\begin{aligned}
& \hat{\bar{Y}}_{R}=\frac{\bar{y}}{\bar{x}} \overline{\mathrm{X}}=\hat{R} \bar{X} \\
& \text { Where } \hat{R}=\frac{\bar{y}}{\bar{x}}, \bar{y}=\frac{1}{n} \sum_{i=1}^{n} y_{i}, \bar{x}=\frac{1}{n} \sum_{i=1}^{n} x_{i}
\end{aligned}
$$

the bias and mean squared error of ratio estimator up to first order approximations are

$$
\begin{aligned}
& \mathrm{B}\left(\hat{\bar{Y}}_{R}\right)=\left(\frac{1-\mathrm{f}}{\mathrm{n}}\right) \bar{Y}\left[C_{x}{ }^{2}-\rho C_{x} C_{y}\right] \\
& \operatorname{MSE}\left(\hat{\bar{Y}}_{R}\right)=\left(\frac{1-\mathrm{f}}{\mathrm{n}}\right) \bar{Y}^{2}\left[{C_{y}}^{2}+{C_{x}}^{2}-2 \rho C_{x} C_{y}\right]
\end{aligned}
$$

Where $C_{x}=\frac{s_{x}}{\bar{X}}, \rho=\frac{s_{x y}}{S_{x} S_{y}}, S_{x y}=\frac{1}{N-1} \sum_{i=1}^{N}\left(Y_{i}-\bar{Y}\right)\left(X_{i}-\bar{X}\right), S_{x}{ }^{2}=\frac{1}{N-1} \sum_{i=1}^{N}\left(X_{i}-\bar{X}\right)^{2}$

The modified ratio estimator for the population mean $\bar{Y}$ with known median of the auxiliary variable is

$$
\hat{\bar{Y}}_{M R}=\bar{y}\left(\frac{\bar{X}+M_{d}}{\bar{x}+M_{d}}\right)
$$

The bias and mean squared error of modified ratio estimator are given by

$$
\begin{aligned}
& \mathrm{B}\left(\hat{\bar{Y}}_{M R}\right)=\left(\frac{1-\mathrm{f}}{\mathrm{n}}\right) \bar{Y}\left[\theta^{2} C_{x}{ }^{2}-\theta \rho \mathrm{C}_{\mathrm{x}} \mathrm{C}_{\mathrm{y}}\right] \\
& \operatorname{MSE}\left(\hat{\bar{Y}}_{M R}\right)=\left(\frac{1-\mathrm{f}}{\mathrm{n}}\right) \bar{Y}^{2}\left[C_{y}{ }^{2}+\theta^{2} C_{x}{ }^{2}-2 \theta \rho \mathrm{C}_{\mathrm{x}} \mathrm{C}_{\mathrm{y}}\right]
\end{aligned}
$$

where $\theta=\frac{\bar{X}}{\bar{X}+M_{d}}$

The auxiliary variable and study variable are negatively correlated, the product estimator and its modifications are used. The product estimator (Murthy, 1964) is given by

$$
\hat{\bar{Y}}_{p}=\bar{y} \frac{\bar{x}}{\bar{x}} \text {. }
$$

The bias and mean squared error of the product estimator are given by

$$
\begin{aligned}
& \mathrm{B}\left(\hat{\bar{Y}}_{p}\right) \quad=\left(\frac{1-\mathrm{f}}{\mathrm{n}}\right) \bar{Y}\left[\rho \mathrm{C}_{\mathrm{x}} \mathrm{C}_{\mathrm{y}}\right] \\
& \operatorname{MSE}\left(\hat{\bar{Y}}_{p}\right)=\left(\frac{1-\mathrm{f}}{\mathrm{n}}\right) \bar{Y}^{2}\left[C_{y}{ }^{2}+C_{x}{ }^{2}+2 \rho \mathrm{C}_{\mathrm{x}} \mathrm{C}_{\mathrm{y}}\right]
\end{aligned}
$$

The modified product estimator with known median of the auxiliary variable is defined by 


$$
\hat{\bar{Y}}_{M p}=\bar{y}\left(\frac{\bar{x}+M_{d}}{\bar{X}+M_{d}}\right)
$$

The bias and mean squared error of the modified product estimator are given by

$$
\begin{aligned}
\mathrm{B}\left(\hat{\bar{Y}}_{M p}\right) & =\left(\frac{1-\mathrm{f}}{\mathrm{n}}\right) \bar{Y}\left[\theta \rho C_{x} C_{y}\right] \\
\operatorname{MSE}\left(\hat{\bar{Y}}_{M p}\right) & =\left(\frac{1-\mathrm{f}}{\mathrm{n}}\right) \bar{Y}^{2}\left[C_{y}{ }^{2}+\theta^{2} C_{x}{ }^{2}+2 \theta \rho \mathrm{C}_{\mathrm{x}} \mathrm{C}_{\mathrm{y}}\right]
\end{aligned}
$$

where $\theta=\frac{\bar{X}}{\bar{X}+M_{d}}$

\section{RESULTS}

In this section, a ratio cum product estimator for the population mean by using the known median of an auxiliary variable is proposed and also derived the bias and the mean squared errors of the proposed estimators. Compare the mean squared error of the proposed estimator with the above mentioned estimators by algebraically and numerically with the help of three natural populations. The detailed expressions are given below

\section{Proposed estimator}

The proposed ratio cum product estimator for the population mean by using the known population median of the auxiliary variable is given by

$$
\begin{aligned}
& \widehat{Y}_{p r}=\alpha \lambda_{1} \bar{y}\left(\frac{\bar{X}+M_{d}}{\bar{x}+M_{d}}\right)+(1-\alpha) \lambda_{2} \bar{y}\left(\frac{\bar{x}+M_{d}}{\bar{X}+M_{d}}\right) \\
& \text { where } \lambda_{1}=\frac{s_{y}}{s_{y}+K_{1} C_{y}} \lambda_{2}=\frac{s_{y}}{s_{y}+K_{2} C_{y}} \text { where } K_{1} \text { and } K_{2} \text { are constants }
\end{aligned}
$$

\section{The bias and mean squared error of the proposed estimator}

To obtain the bias and mean squared error of the proposed estimator,

Consider $e_{0}=\frac{\bar{y}-\bar{Y}}{\bar{Y}}, e_{1}=\frac{\bar{x}-\bar{X}}{\bar{X}}, \theta=\frac{\bar{X}}{\bar{X}+M_{d}}$

$$
E\left(e_{0}\right)=E\left(e_{1}\right)=0, E\left(e_{0}^{2}\right)=\left(\frac{1-f}{n}\right) \bar{Y}^{2} C_{y}{ }^{2}, E\left(e_{1}^{2}\right)=\left(\frac{1-f}{n}\right) \bar{X}^{2} C_{x}{ }^{2}, E\left(e_{0} e_{1}\right)=\left(\frac{1-f}{n}\right) \rho C_{x} C_{y}
$$

Substitute the values of $e_{0}$ and $e_{1}$ in equation (6) and neglecting the high order expressions, we get

$$
\begin{gathered}
B\left(\hat{\bar{Y}}_{P r}\right)=E\left(\hat{\bar{Y}}_{P r}-\bar{Y}\right) \\
B\left(\hat{\bar{Y}}_{P r}\right)=\bar{Y}\left(\alpha \lambda_{1}+(1-\alpha) \lambda_{2}-1\right)+\left(\frac{1-f}{n}\right) \bar{Y}\left\{\alpha \lambda_{1} \theta^{2} C_{x}{ }^{2}-\theta \rho C_{x} C_{y}\left(\alpha \lambda_{1}-(1-\alpha) \lambda_{2}\right)\right\}
\end{gathered}
$$

The detailed derivation of the mean squared error is given in the appendix and the final expression is obtained with only first order approximation in the Taylor series expansion as

$\operatorname{MSE}\left(\hat{\bar{Y}}_{P r}\right)=E\left(\hat{\bar{Y}}_{P r}-\bar{Y}\right)^{2}$ 


$$
\begin{aligned}
\operatorname{MSE}\left(\hat{\bar{Y}}_{P r}\right)=\bar{Y}^{2}( & \left.\alpha \lambda_{1}+(1-\alpha) \lambda_{2}-1\right)^{2}+\left(\frac{1-f}{n}\right) \bar{Y}^{2}\left\{C_{y}{ }^{2}\left(\alpha \lambda_{1}+(1-\alpha) \lambda_{2}\right)^{2}\right. \\
& +\theta^{2} C_{x}{ }^{2}\left(3 \alpha^{2} \lambda_{1}{ }^{2}+(1-\alpha)^{2} \lambda_{2}{ }^{2}-2 \alpha \lambda_{1}\right)+2 \theta \rho C_{x} C_{y}\left(\alpha \lambda_{1}-(1-\alpha) \lambda_{2}\right) \\
& \left.-2\left(\alpha^{2} \lambda_{1}{ }^{2}-(1-\alpha)^{2} \lambda_{2}{ }^{2}\right)\right\}
\end{aligned}
$$

where $\lambda_{1}$ and $\lambda_{2}$ are as defined above. If we assume that $K_{1}=0, K_{2}=0$ and $\alpha=1$ then the proposed estimator are exactly equal to the existing modified ratio estimator and if $K_{1}=0, K_{2}=$ 0 and $\alpha=0$ then the proposed estimator is exactly equal to the existing modified product estimator we discussed above. If we assume that $K_{1}=B\left(\hat{\bar{Y}}_{M R}\right), K_{2}=B\left(\hat{Y}_{M P}\right)$ then the proposed estimators are almost unbiased ratio cum product estimator under optimum values of $\alpha$. The optimal value of $\alpha$ is determined by minimizing the MSE $\left(\hat{\bar{Y}}_{p r}\right)$ with respect to $\alpha$. For this differentiate MSE with respect to $\alpha$ and equate to zero.

$$
\begin{gathered}
\frac{\partial M S E}{\partial \alpha}=0, \text { and we get the value of } \alpha, \text { as } \\
\alpha=\frac{\left(\lambda_{2}-1\right)\left(\lambda_{2}-\lambda_{1}\right)+\left(\frac{1-f}{n}\right)\left\{C_{y}{ }^{2} \lambda_{2}\left(\lambda_{2}-\lambda_{1}\right)+\theta^{2} C_{x}{ }^{2}\left(\lambda_{1}+\lambda_{2}{ }^{2}\right)-\theta \rho C_{x} C_{y}\left(\lambda_{1}+\lambda_{2}-4 \lambda_{2}{ }^{2}\right)\right\}}{\left(\lambda_{1}-\lambda_{2}\right)^{2}+\left(\frac{1-f}{n}\right)\left\{\left(\lambda_{1}-\lambda_{2}\right)^{2} C_{y}{ }^{2}+\theta^{2} C_{x}{ }^{2}\left(3 \lambda_{1}{ }^{2}+\lambda_{2}{ }^{2}\right)+4 \theta \rho C_{x} C_{y}\left(\lambda_{2}{ }^{2}-\lambda_{1}{ }^{2}\right)\right\}}
\end{gathered}
$$

\section{Efficiency comparison}

To assess the efficiencies of the proposed estimator with that of the estimators we discussed above, the mean squared error under optimality condition of these estimators are used and it satisfies the following cases. The proposed estimator under optimality condition is more efficient than of existing estimators such as

$$
V\left(\bar{y}_{s r s}\right), \operatorname{MSE}\left(\hat{\bar{Y}}_{R}\right), \operatorname{MSE}\left(\hat{\bar{Y}}_{M R}\right), \operatorname{MSE}\left(\hat{\bar{Y}}_{P}\right), \operatorname{MSE}\left(\hat{\bar{Y}}_{M P}\right),
$$

and it satisfying the following conditions.

Case (1) $V\left(\bar{y}_{\text {srs }}\right) \geq \operatorname{MSE}\left(\hat{\bar{Y}}_{P r}\right)$

$$
\rho \geq \frac{(A-1)^{2}+\left(\frac{1-f}{n}\right)\left\{\left(A^{2}-1\right) C_{y}{ }^{2}+\theta^{2} C_{x}{ }^{2}\left(A^{2}+(A+B)(B-1)\right)\right\}}{\left(\frac{1-f}{n}\right) 2 \theta C_{x} C_{y}(B(2 A-1)}
$$

Case (2) $\operatorname{MSE}\left(\hat{\bar{Y}}_{R}\right) \geq \operatorname{MSE}\left(\hat{\bar{Y}}_{P r}\right)$

$$
\rho \geq \frac{(A-1)^{2}+\left(\frac{1-f}{n}\right)\left\{\left(A^{2}-1\right) C_{y}{ }^{2}+C_{x}{ }^{2}\left(\theta^{2}\left(A^{2}+(A+B)(B-1)\right)-1\right)\right\}}{\left(\frac{1-f}{n}\right) 2 C_{x} C_{y}(\theta B(2 A-1)-1)}
$$

Case (3) $\operatorname{MSE}\left(\hat{\bar{Y}}_{M R}\right) \geq \operatorname{MSE}\left(\hat{\bar{Y}}_{P r}\right)$ 


$$
\rho \geq \frac{(A-1)^{2}+\left(\frac{1-f}{n}\right)\left\{\left(A^{2}-1\right) C_{y}{ }^{2}+\theta^{2} C_{x}{ }^{2}\left(\left(A^{2}+(A+B)(B-1)\right)-1\right)\right\}}{\left(\frac{1-f}{n}\right) 2 \theta C_{x} C_{y}(B(2 A-1)-1)}
$$

Case (4) $\operatorname{MSE}\left(\hat{\bar{Y}}_{P}\right) \geq \operatorname{MSE}\left(\hat{\bar{Y}}_{P r}\right)$

$$
\rho \geq \frac{(A-1)^{2}+\left(\frac{1-f}{n}\right)\left\{\left(A^{2}-1\right) C_{y}{ }^{2}+C_{x}{ }^{2}\left(\theta^{2}\left(A^{2}+(A+B)(B-1)\right)-1\right)\right\}}{\left(\frac{1-f}{n}\right) 2 C_{x} C_{y}(\theta B(2 A-1)+1)}
$$

Case (5) $\operatorname{MSE}\left(\hat{\bar{Y}}_{M P}\right) \geq \operatorname{MSE}\left(\hat{\bar{Y}}_{P r}\right)$

$$
\rho \geq \frac{(A-1)^{2}+\left(\frac{1-f}{n}\right)\left\{\left(A^{2}-1\right) C_{y}{ }^{2}+\theta^{2} C_{x}{ }^{2}\left(\left(A^{2}+(A+B)(B-1)\right)-1\right)\right\}}{\left(\frac{1-f}{n}\right) 2 \theta C_{x} C_{y}(B(2 A-1)+1)}
$$

where $A=\alpha \lambda_{1}+(1-\alpha) \lambda_{2}$ and $B=\alpha \lambda_{1}-(1-\alpha) \lambda_{2}$

\section{Numerical study}

In this section we consider four natural populations, the computed values of constants and parameters of these populations are given below

Population 1: (Khoshnevisan, Singh, Chauhan, Sawan and Smarandache, 2007)

$$
\begin{array}{llllllll}
N=80 & n=5 & \bar{Y}=5182.562 & \bar{X}=1126.463 & \rho=0.9413 & S_{\mathrm{x}}=845.609 & S_{\mathrm{y}}=1835.654 \\
C_{\mathrm{x}}=0.750 & C_{\mathrm{y}}=0.354 & \beta_{1}=1.061 & \beta_{2}=2.866 & \alpha=0.867 & \theta=0.597 & \lambda_{1}=0.990 & \lambda_{2}=0 .
\end{array}
$$

Population 2: (Singh and Chaudhary, 1986)

$$
\begin{array}{lllllll}
N=34 & n=5 & \bar{Y}=199.441 & \bar{X}=856.411 & \rho=0.445 & S_{\mathrm{x}}=733.140 & S_{\mathrm{y}}=150.215 \\
C_{\mathrm{x}}=0.856 & C_{\mathrm{y}}=0.753 & \beta_{1}=7.955 & \beta_{2}=13.366 & \alpha=0.858 & \theta=0.527 & \lambda_{1}=0.991 \\
\lambda_{2}=0.974 & & & & & &
\end{array}
$$

Population 3: (Cochran, 1977)

$$
\begin{array}{lllllll}
N=49 & n=5 & \bar{Y}=127.796 & \bar{X}=103.143 & \rho=0.982 & S_{\mathrm{x}}=104.405 & S_{y}=123.121 \\
C_{\mathrm{x}}=1.012 & C_{\mathrm{y}}=0.963 & \beta_{1}=4.777 & \beta_{2}=7.511 & \alpha=1.356 & \theta=0.617 & \lambda_{1}=1.037 \\
\lambda_{2}=0.904 & & & & & & \\
\end{array}
$$

These values are used to obtain the bias and mean squared error of proposed estimator and existing estimators under optimality conditions. The mean square error of the proposed estimator is used to compare the percentage relative efficiency with that of the existing estimators such as the simple random sample mean, ratio, product, modified ratio estimator and the modified product estimator. 
Table 1. Bias and MSE of proposed and existing estimators from different populations

\begin{tabular}{ccccccc}
\hline \multirow{2}{*}{ Estimator } & \multicolumn{2}{c}{ Population 1 } & \multicolumn{2}{c}{ Population 2 } & \multicolumn{2}{c}{ Population 3 } \\
\cline { 2 - 7 } & Bias & MSE & Bias & MSE & Bias & MSE \\
\hline Proposed (opt) & $-4.62 \mathrm{e}-13$ & 66429.21 & $8.88 \mathrm{e}-16$ & 3013.05 & $1.06 \mathrm{e}-14$ & 6.83 \\
\hline $\bar{y}_{\text {srs }}$ & - & 631805.10 & - & 3849.91 & - & 2722.40 \\
\hline$\hat{\bar{Y}}_{R}$ & 304.37 & 948789.10 & 15.16 & 4925.33 & 1.54 & 111.43 \\
\hline$\hat{\bar{Y}}_{p}$ & 243.21 & 5990618.00 & 9.77 & 12718.48 & 21.97 & 11343.93 \\
\hline$\hat{\bar{Y}}_{M R}$ & 50.35 & 139073.90 & 1.78 & 3177.31 & -4.60 & 401.08 \\
\hline$\hat{\bar{Y}}_{M P}$ & 145.42 & 3153694.00 & 5.15 & 7287.23 & 13.56 & 7332.58 \\
\hline
\end{tabular}

Table 2. Percentage relative efficiency of the proposed estimator

\begin{tabular}{cccc}
\hline Estimators & Population 1 & Population 2 & Population 3 \\
\hline $\bar{y}_{s r s}$ & 668.76 & 174.33 & 173.79 \\
\hline$\hat{Y}_{R}$ & 2858.75 & 109.76 & 222.37 \\
\hline$\hat{\bar{Y}}_{p}$ & 127.04 & 511.41 & 574.21 \\
\hline$\hat{\bar{Y}}_{M R}$ & 2452.84 & 109.48 & 219.87 \\
\hline$\hat{Y}_{M P}$ & 103.65 & 509.51 & 568.48 \\
\hline
\end{tabular}

\section{CONCLUSION}

The performance of the proposed estimator for some known natural populations is also observed. when $K_{1}=B\left(\hat{\bar{Y}}_{M R}\right), K_{2}=B\left(\hat{\bar{Y}}_{M P}\right)$ and $\alpha$ is optimum the proposed estimator is less bias (almost unbiased ) than the existing estimators and it is more efficient than all these existing estimators. In the fact the percentage relative efficiency is ranging from

- In the case of simple random sampling, the percentage relative efficiency ranged from 173.79 to 668.76.

- In the case of ratio and modified ratio estimators, the PRE is ranged from 109.76 to 2858.75 and 109.48 and 2452.84

- In case of product and modified product estimators, the percentage relative efficiencies are ranged from 127.04 to 574.21 and 103.65 to 568.48 
It shows that proposed estimator performing better than all these existing estimators.

\section{CONFLICT OF INTEREST}

The authors declared that there is no conflict of interest.

\section{REFERENCES}

Cochran, W.G. (1940). The estimation of the yields of the cereal experiments by sampling for the ratio of grain to total produce. The Journal of Agricultural Science, 30, 262-275.

Cochran, W. G. (1977). Sampling Techniques ( ${ }^{\text {rd }}$ ed.). Wiley Eastern Limited.

Kadilar, C., \& Cingi, H. (2004). Ratio estimators in simple random sampling. Appl Math Comput, 151, 893-902.

Kadilar, C., \& Cingi, H. (2006). Improvement in estimating the population means in simple random sampling. Appl Math Comput, 19, 75-79.

Khoshnevisan, M., Singh, R., Chauhan, P., Sawan, N., \& Smarandache, F. (2007). A general family of estimators for estimating population mean using known value of some population parameter(s). Far East Journal of Theoretical Statistics, 22,181-191.

Murthy, M.N. (1964). Product method of estimation. Sankhya A, 26, 69-74.

Murthy, M.N. (1967). Sampling theory and methods. Statistical Publishing Society, Calcutta, India,

Singh, D., \& Chaudhary, F.S. (1986). Theory and analysis of sample survey designs, New Age International Publisher.

Singh, H. P., Tailor, R., \& Kakran, M. S. (2004): An improved estimator of the population mean using power transformation. Journal of the Indian Society of Agricultural Statistics, 58(2), 223-230.

Sisodia, B.V.S, \& Dwivedi, V. K. (1981). A modified ratio estimator using the coefficient of variation of auxiliary variable. Jour. Ind. Soc. Agri. Stat., 33(1), 13-18.

Subramani, J. (2013). Generalized modified ratio estimator for estimation of finite population mean. Journal of Modern Applied Statistical Methods, 12, 121-155.

Subramani, J., \& Master Ajith S. (2016a). Improved ratio cum product estimator with known coefficient of variation in simple random sampling. J. Adv. Res. Appl. Math. Stat., 1(2), 6070.

Subramani, J., \& Master Ajith S. (2016b). Modified ratio cum product estimators for estimation of finite population mean with known correlation coefficient. Biom Biostat Int J, 4(6), 00113. http://dx.doi.org/10.15406/bbij.2016.04.00113 
Subramani, J., \& Kumarapandiyan, G. (2012a). Modified ratio estimators for population mean using function of quartiles of auxiliary variable. Bonfring International Journal of Industrial Engineering and Management Science, 2 (2), 19-23.

Subramani, J., \& Kumarapandiyan, G. (2012b). Variance estimation using quartiles and their functionsof an auxiliary variable. International Journal of Statistics and Applications, 2 (5), $67-72$.

Upadhyaya, L.N., \& Singh, H.P. (1999). Use of transformed auxiliary variable in estimating the finite population mean. Biometrical Journal, 41 (5), 627-636.

Yan, Z., \& Tian, B. (2010). Ratio method to the mean estimation using co-efficient of skewness of auxiliary variable. International Conference on Information Computing and Applications, China, Part II, CCIS 106, 103-110.

Reference to this paper should be made as follows:

Subramani, J., \& Master Ajith S. (2017). Almost unbiased ratio cum product estimator for finite population mean with known median in simple random sampling. Nep. J. Stat., 1, 1-14. 


\section{APPENDIX}

\section{Bias and MSE of Proposed Estimators}

The proposed ratio cum product estimator is given by

$$
\widehat{\bar{Y}}_{p r}=\alpha \lambda_{1} \bar{y}\left(\frac{\bar{x}+\mathrm{M}_{\mathrm{d}}}{\bar{x}+\mathrm{M}_{\mathrm{d}}}\right)+(1-\alpha) \lambda_{2} \bar{y}\left(\frac{\bar{x}+\mathrm{M}_{\mathrm{d}}}{\overline{\bar{x}}+\mathrm{M}_{\mathrm{d}}}\right)
$$

here $\lambda_{1}=\frac{s_{y}}{s_{y}+K_{1} C_{y}}$ and $\lambda_{2}=\frac{s_{y}}{s_{y}+K_{2} C_{y}}, K_{1}, K_{2}$ are constants

To obtain the bias and mean squared error of the proposed estimator,

Consider, $\quad e_{0}=\frac{\bar{y}-\bar{Y}}{\bar{Y}}, e_{1}=\frac{\bar{x}-\bar{X}}{\bar{X}}, \theta=\frac{\bar{X}}{\bar{X}+\mathrm{M}_{\mathrm{d}}}$

$E\left(e_{0}\right)=E\left(e_{1}\right)=0, E\left(e_{0}{ }^{2}\right)=\left(\frac{1-f}{n}\right) \bar{Y}^{2} C_{y}{ }^{2}, E\left(e_{1}{ }^{2}\right)=\left(\frac{1-f}{n}\right) \bar{X}^{2} C_{x}{ }^{2}, E\left(e_{0} e_{1}\right)=\left(\frac{1-f}{n}\right) \mathrm{C}_{\mathrm{xy}}$

Substitute these values in $\widehat{\bar{Y}}_{p r}$ and neglecting the high order expressions, we get

$$
\begin{aligned}
\widehat{\bar{Y}}_{p r} & =\alpha \lambda_{1} \bar{y}\left(\frac{\bar{X}+\mathrm{M}_{\mathrm{d}}}{\bar{x}+\mathrm{M}_{\mathrm{d}}}\right)+(1-\alpha) \lambda_{2} \bar{y}\left(\frac{\bar{x}+\mathrm{M}_{\mathrm{d}}}{\bar{X}+\mathrm{M}_{\mathrm{d}}}\right) \\
& =\alpha \lambda_{1} \bar{Y}\left(1+e_{0}\right)\left(1+\theta e_{1}\right)^{-1}+(1-\alpha) \lambda_{2} \bar{Y}\left(1+e_{0}\right)\left(1+\theta e_{1}\right) \\
& =\bar{Y}\left\{\alpha \lambda_{1}\left(1+e_{0}\right)\left(1-\theta e_{1}+\theta^{2} e_{1}{ }^{2}\right)+(1-\alpha) \lambda_{2}\left(1+e_{0}\right)\left(1+\theta e_{1}\right)\right\} \\
& =\bar{Y}\left\{\alpha \lambda_{1}\left(1-\theta e_{1}+\theta^{2} e_{1}{ }^{2}+e_{0}-\theta e_{1} e_{0}\right)+(1-\alpha) \lambda_{2}\left(1+\theta e_{1}+e_{0}+\theta e_{0} e_{1}\right)\right\} \\
\widehat{\bar{Y}}_{p r}-\bar{Y}= & \bar{Y}\left\{\alpha \lambda_{1}\left(1-\theta e_{1}+\theta^{2} e_{1}{ }^{2}+e_{0}-\theta e_{1} e_{0}\right)+(1-\alpha) \lambda_{2}\left(1+\theta e_{1}+e_{0}+\theta e_{0} e_{1}\right)\right\} \\
B\left(\hat{\bar{Y}}_{P r}\right)= & E\left(\hat{\bar{Y}}_{P r}-\bar{Y}\right) \\
& =E\left\{\bar{Y}\left(\alpha \lambda_{1}\left(1-\theta e_{1}+\theta^{2} e_{1}{ }^{2}+e_{0}-\theta e_{1} e_{0}\right)+(1-\alpha) \lambda_{2}\left(1+\theta e_{1}+e_{0}+\theta e_{0} e_{1}\right)-1\right)\right\} \\
= & \bar{Y}\left\{\alpha \lambda_{1}\left(1+\left(\frac{1-f}{n}\right) \theta^{2} C_{x}{ }^{2}-\left(\frac{1-f}{n}\right) \theta \mathrm{C}_{\mathrm{xy}}\right)+(1-\alpha) \lambda_{2}\left(1+\left(\frac{1-f}{n}\right) \theta \mathrm{C}_{\mathrm{xy}}\right)-1\right\} \\
B\left(\hat{\bar{Y}}_{P r}\right)= & \bar{Y}\left\{\left(\alpha \lambda_{1}+(1-\alpha) \lambda_{2}-1\right)+\left(\frac{1-f}{n}\right)\left\{\alpha \lambda_{1} \theta^{2} C_{x}{ }^{2}-\theta \mathrm{C}_{\mathrm{xy}}\left(\alpha \lambda_{1}-(1-\alpha) \lambda_{2}\right)\right\}\right\}
\end{aligned}
$$

The mean squared error of the proposed estimator is

$$
\begin{aligned}
& \operatorname{MSE}\left(\hat{\bar{Y}}_{P r}\right)=E\left(\hat{\bar{Y}}_{P r}-\bar{Y}\right)^{2} \\
& =E\left\{\bar{Y}\left(\alpha \lambda_{1}\left(1-\theta e_{1}+\theta^{2} e_{1}^{2}+e_{0}-\theta e_{1} e_{0}\right)+(1-\alpha) \lambda_{2}\left(1+\theta e_{1}+e_{0}+\theta e_{0} e_{1}\right)-1\right)^{2}\right\} \\
& =E\left\{\overline { Y } ^ { 2 } \left(\alpha^{2} \lambda_{1}^{2}\left(1-\theta e_{1}+\theta^{2} e_{1}^{2}+e_{0}-\theta e_{1} e_{0}\right)^{2}+(1-\alpha)^{2} \lambda_{2}{ }^{2}\left(1+\theta e_{1}+e_{0}+\theta e_{0} e_{1}\right)^{2}+1+\right.\right. \\
& 2 \alpha \lambda_{1}(1-\alpha) \lambda_{2}\left(1-\theta e_{1}+\theta^{2}{e_{1}}^{2}+e_{0}-\theta e_{1} e_{0}\right)\left(1+\theta e_{1}+e_{0}+\theta e_{0} e_{1}\right)-2 \alpha \lambda_{1}\left(1-\theta e_{1}+\right. \\
& \left.\left.\theta^{2} e_{1}{ }^{2}+e_{0}-\theta e_{1} e_{0}\right)-2(1-\alpha) \lambda_{2}\left(1+\theta e_{1}+e_{0}+\theta e_{0} e_{1}\right)\right\}
\end{aligned}
$$




$$
\begin{gathered}
=\bar{Y}^{2}\left\{\alpha^{2} \lambda_{1}{ }^{2}\left(1+3\left(\frac{1-f}{n}\right) \theta^{2} C_{x}{ }^{2}+\left(\frac{1-f}{n}\right) C_{y}{ }^{2}-4\left(\frac{1-f}{n}\right) \theta \mathrm{C}_{\mathrm{xy}}\right)+(1-\alpha)^{2} \lambda_{2}{ }^{2}\left(1+\left(\frac{1-f}{n}\right) \theta^{2} C_{x}{ }^{2}+\right.\right. \\
\left.\left(\frac{1-f}{n}\right) C_{y}{ }^{2}+\left(\frac{1-f}{n}\right) 4 \theta \mathrm{C}_{\mathrm{xy}}\right)+2 \alpha \lambda_{1}(1-\alpha) \lambda_{2}\left(1+\left(\frac{1-f}{n}\right) C_{y}{ }^{2}\right)-2 \alpha \lambda_{1}\left(1+\left(\frac{1-f}{n}\right) \theta^{2} C_{x}{ }^{2}-\right. \\
\left.\left.\left(\frac{1-f}{n}\right) \theta \mathrm{C}_{\mathrm{xy}}\right)-2(1-\alpha) \lambda_{2}\left(1+\left(\frac{1-f}{n}\right) \theta \mathrm{C}_{\mathrm{xy}}\right)\right\} \\
\operatorname{MSE}\left(\hat{\bar{Y}}_{P r}\right)=\bar{Y}^{2}\left\{\left(\alpha \lambda_{1}+(1-\alpha) \lambda_{2}-1\right)^{2}+\left(\frac{1-f}{n}\right)\left\{C_{y}{ }^{2}\left(\alpha \lambda_{1}+(1-\alpha) \lambda_{2}\right)^{2}\right.\right. \\
+\theta^{2} C_{x}{ }^{2}\left(3 \alpha^{2} \lambda_{1}{ }^{2}+(1-\alpha)^{2} \lambda_{2}{ }^{2}-2 \alpha \lambda_{1}\right)+2 \theta \mathrm{C}_{\mathrm{xy}}\left(\alpha \lambda_{1}-(1-\alpha) \lambda_{2}\right) \\
\left.\left.-2\left(\alpha^{2} \lambda_{1}{ }^{2}-(1-\alpha)^{2} \lambda_{2}{ }^{2}\right)\right\}\right\}
\end{gathered}
$$

Where $\quad \theta=\frac{\bar{X}}{\bar{X}+M_{d}}$

The optimal value of $\alpha$ is determined by minimize the MSE $\left(\hat{\bar{Y}}_{p r}\right)$ with respect to $\alpha$. For this differentiate MSE with respect to $\alpha$ and equate to zero.

$$
\begin{aligned}
& \text { ie, } \frac{\partial M S E}{\partial \alpha}=0, \text { and we get the value of } \alpha, \text { as } \\
& 2\left(\alpha \lambda_{1}+(1-\alpha) \lambda_{2}-1\right)\left(\lambda_{1}-\lambda_{2}\right)+\left(\frac{1-f}{n}\right)\left\{\mathrm{C}_{\mathrm{y}}{ }^{2} 2\left(\alpha \lambda_{1}+(1-\alpha) \lambda_{2}\right)\left(\lambda_{1}-\lambda_{2}\right)+\theta^{2} \mathrm{C}_{\mathrm{x}}{ }^{2}\left(6 \alpha \lambda_{1}{ }^{2}-\right.\right. \\
& \left.2(1-\alpha) \lambda_{2}{ }^{2}-2 \lambda_{1}\right)+2 \theta \mathrm{C}_{\mathrm{xy}}\left(\lambda_{1}+\lambda_{2}-2\left(2 \alpha \lambda_{1}{ }^{2}+2(1-\alpha) \lambda_{2}{ }^{2}\right)\right\}=0 \\
& \quad\left(\alpha \lambda_{1}-\alpha \lambda_{2}\right)\left(\lambda_{1}-\lambda_{2}\right)+\left(\frac{1-f}{n}\right)\left\{\mathrm{C}_{\mathrm{y}}{ }^{2}\left(\alpha \lambda_{1}-\alpha \lambda_{2}\right)\left(\lambda_{1}-\lambda_{2}\right)+\theta^{2} \mathrm{C}_{\mathrm{x}}{ }^{2}\left(3 \alpha \lambda_{1}{ }^{2}+\alpha \lambda_{2}{ }^{2}\right)+\right. \\
& \left.\theta \mathrm{C}_{\mathrm{xy}}\left(-4 \alpha \lambda_{1}{ }^{2}+4 \alpha \lambda_{2}{ }^{2}\right)\right\}=\left(\lambda_{2}-1\right)\left(\lambda_{2}-\lambda_{1}\right)+\left(\frac{1-f}{n}\right)\left\{\mathrm{C}_{\mathrm{y}}{ }^{2} \lambda_{2}\left(\lambda_{2}-\lambda_{1}\right)+\theta^{2} \mathrm{C}_{\mathrm{x}}{ }^{2}\left(\lambda_{1}+\lambda_{2}{ }^{2}\right)-\right. \\
& \left.\theta \mathrm{C}_{\mathrm{xy}}\left(\lambda_{1}+\lambda_{2}-4 \lambda_{2}{ }^{2}\right)\right\} \\
& \alpha=\frac{\left(\lambda_{2}-1\right)\left(\lambda_{2}-\lambda_{1}\right)+\left(\frac{1-f}{n}\right)\left\{C_{y}{ }^{2} \lambda_{2}\left(\lambda_{2}-\lambda_{1}\right)+\theta^{2} C_{x}{ }^{2}\left(\lambda_{1}+\lambda_{2}{ }^{2}\right)-\theta \mathrm{C}_{\mathrm{xy}}\left(\lambda_{1}+\lambda_{2}-4 \lambda_{2}{ }^{2}\right)\right\}}{\left(\lambda_{1}-\lambda_{2}\right)^{2}+\left(\frac{1-f}{n}\right)\left\{\left(\lambda_{1}-\lambda_{2}\right)^{2} C_{y}{ }^{2}+\theta^{2} C_{x}{ }^{2}\left(3 \lambda_{1}{ }^{2}+\lambda_{2}{ }^{2}\right)+4 \theta \mathrm{C}_{\mathrm{xy}}\left(\lambda_{2}{ }^{2}-\lambda_{1}{ }^{2}\right)\right\}}
\end{aligned}
$$

Theorem:

The proposed estimator under optimality condition is more efficient than of existing estimators such as

$$
V\left(\bar{y}_{s r s}\right), \operatorname{MSE}\left(\hat{\bar{Y}}_{R}\right), \operatorname{MSE}\left(\hat{\bar{Y}}_{M R}\right), \operatorname{MSE}\left(\hat{\bar{Y}}_{P}\right), \operatorname{MSE}\left(\hat{\bar{Y}}_{M P}\right)
$$

and it satisfying the following condition

Proof:

Case (1) : The proposed estimator is more efficient than simple random sampling without replacement estimator if

$$
V\left(\bar{y}_{s r S}\right)-\operatorname{MSE}\left(\hat{\bar{Y}}_{P r}\right) \geq 0
$$




$$
\begin{gathered}
\left(\frac{1-f}{n}\right) \bar{Y}^{2} C_{y}{ }^{2}-\bar{Y}^{2}\left(\left(\alpha \lambda_{1}+(1-\alpha) \lambda_{2}-1\right)^{2}+\left(\frac{1-f}{n}\right)\left\{C_{y}{ }^{2}\left(\alpha \lambda_{1}+(1-\alpha) \lambda_{2}\right)^{2}+\theta^{2} C_{x}{ }^{2}\left(3 \alpha^{2} \lambda_{1}{ }^{2}+\right.\right.\right. \\
\left.\left.(1-\alpha)^{2} \lambda_{2}{ }^{2}-2 \alpha \lambda_{1}\right)+2 \theta C_{\mathrm{xy}}\left(\alpha \lambda_{1}-(1-\alpha) \lambda_{2}-2\left(\alpha^{2} \lambda_{1}{ }^{2}-(1-\alpha)^{2} \lambda_{2}{ }^{2}\right)\right\}\right) \geq 0 \\
\left(\frac{1-f}{n}\right) \bar{Y}^{2} C_{y}{ }^{2}\left(1-\left(\alpha \lambda_{1}+(1-\alpha) \lambda_{2}\right)^{2}\right) \geq \bar{Y}^{2}\left(\left(\alpha \lambda_{1}+(1-\alpha) \lambda_{2}-1\right)^{2}+\left(\frac{1-f}{n}\right)\left\{\theta ^ { 2 } C _ { x } { } ^ { 2 } \left(3 \alpha^{2} \lambda_{1}{ }^{2}+\right.\right.\right. \\
\left.\left.(1-\alpha)^{2} \lambda_{2}{ }^{2}-2 \alpha \lambda_{1}\right)+2 \theta \mathrm{C}_{\mathrm{xy}}\left(\alpha \lambda_{1}-(1-\alpha) \lambda_{2}-2\left(\alpha^{2} \lambda_{1}{ }^{2}-(1-\alpha)^{2} \lambda_{2}{ }^{2}\right)\right\}\right) \\
\rho \geq \frac{(A-1)^{2}+\left(\frac{1-f}{n}\right)\left\{\left(A^{2}-1\right) C_{y}{ }^{2}+\theta^{2} C_{x}{ }^{2}\left(A^{2}+(A+B)(B-1)\right)\right\}}{\left(\frac{1-f}{n}\right) 2 \theta C_{x} C_{y}(B(2 A-1)}
\end{gathered}
$$

Case (2): The proposed estimator is more efficient than ratio estimator if

$$
\begin{gathered}
V\left(\hat{\bar{Y}}_{R}\right)-\operatorname{MSE}\left(\hat{\bar{Y}}_{P r}\right) \geq 0 \\
\left(\frac{1-f}{n}\right) \bar{Y}^{2}\left(C_{y}{ }^{2}+C_{x}{ }^{2}-2 \mathrm{C}_{\mathrm{xy}}\right)-\bar{Y}^{2}\left(\left(\alpha \lambda_{1}+(1-\alpha) \lambda_{2}-1\right)^{2}+\left(\frac{1-f}{n}\right)\left\{C _ { y } { } ^ { 2 } \left(\alpha \lambda_{1}+\right.\right.\right. \\
\left.(1-\alpha) \lambda_{2}\right)^{2}+\theta^{2} C_{x}{ }^{2}\left(3 \alpha^{2} \lambda_{1}{ }^{2}+(1-\alpha)^{2} \lambda_{2}{ }^{2}-2 \alpha \lambda_{1}\right)+2 \theta \mathrm{C}_{\mathrm{xy}}\left(\alpha \lambda_{1}-(1-\alpha) \lambda_{2}-\right. \\
\left.\left.2\left(\alpha^{2}{\lambda_{1}}^{2}-(1-\alpha)^{2} \lambda_{2}{ }^{2}\right)\right\}\right) \geq 0 \\
\left(\frac{1-f}{n}\right) C_{y}{ }^{2}\left(1-\left(\alpha \lambda_{1}+(1-\alpha) \lambda_{2}\right)^{2}\right) \geq\left(\left(\alpha \lambda_{1}+(1-\alpha) \lambda_{2}-1\right)^{2}+\right. \\
\left(\frac{1-f}{n}\right)\left\{C_{x}{ }^{2}\left(\theta^{2}\left(3 \alpha^{2} \lambda_{1}{ }^{2}+(1-\alpha)^{2} \lambda_{2}{ }^{2}-2 \alpha \lambda_{1}\right)-1\right)+2 \mathrm{C}_{\mathrm{xy}}\left(\theta \left(\alpha \lambda_{1}-(1-\alpha) \lambda_{2}-\right.\right.\right. \\
\left.\left.\left.\left.2\left(\alpha^{2} \lambda_{1}{ }^{2}-(1-\alpha)^{2} \lambda_{2}{ }^{2}\right)\right)+1\right)\right\}\right) \quad\left(\frac{1-f}{n}\right) 2 C_{x} C_{y}(\theta B(2 A-1)-1) \\
\rho \geq \frac{(A-1)^{2}+\left(\frac{1-f}{n}\right)\left\{\left(A^{2}-1\right) C_{y}{ }^{2}+C_{x}{ }^{2}\left(\theta^{2}\left(A^{2}+(A+B)(B-1)\right)-1\right)\right\}}{(1-1)}
\end{gathered}
$$

Case (3): The proposed estimator is more efficient than modified ratio estimator if,

$$
\begin{gathered}
V\left(\hat{\bar{Y}}_{M R}\right)-M S E\left(\hat{\bar{Y}}_{P r}\right) \geq 0 \\
\left(\frac{1-f}{n}\right) \bar{Y}^{2}\left(C_{y}{ }^{2}+\theta^{2} C_{x}{ }^{2}-2 \theta \mathrm{C}_{\mathrm{xy}}\right)-\left(\frac{1-f}{n}\right) \bar{Y}^{2}\left(\left(\alpha \lambda_{1}+(1-\alpha) \lambda_{2}-1\right)^{2}+C_{y}{ }^{2}\left(\alpha \lambda_{1}+\right.\right. \\
\left.(1-\alpha) \lambda_{2}\right)^{2}+\theta^{2} C_{x}{ }^{2}\left(3 \alpha^{2} \lambda_{1}{ }^{2}+(1-\alpha)^{2} \lambda_{2}{ }^{2}-2 \alpha \lambda_{1}\right)+2 \theta \mathrm{C}_{\mathrm{xy}}\left(\alpha \lambda_{1}-(1-\alpha) \lambda_{2}-\right. \\
\left.2\left(\alpha^{2} \lambda_{1}{ }^{2}-(1-\alpha)^{2}{\lambda_{2}}^{2}\right)\right) \geq 0 \\
C_{y}{ }^{2}\left(1-\left(\alpha \lambda_{1}+(1-\alpha) \lambda_{2}\right)^{2}\right) \geq\left(\left(\alpha \lambda_{1}+(1-\alpha) \lambda_{2}-1\right)^{2}+\theta^{2} C_{x}{ }^{2}\left(3 \alpha^{2} \lambda_{1}{ }^{2}+\right.\right. \\
\left.\left.(1-\alpha)^{2} \lambda_{2}{ }^{2}-2 \alpha \lambda_{1}-1\right)+2 \theta \mathrm{C}_{\mathrm{xy}}\left(\alpha \lambda_{1}-(1-\alpha) \lambda_{2}-2\left(\alpha^{2} \lambda_{1}{ }^{2}-(1-\alpha)^{2} \lambda_{2}{ }^{2}\right)+1\right)\right) \\
\left(\frac{1-f}{n}\right) 2 \theta C_{x} C_{y}(B(2 A-1)-1)
\end{gathered}
$$

Case (4): The proposed estimator is more efficient than product estimator if 


$$
\begin{gathered}
V\left(\hat{\bar{Y}}_{P}\right)-\operatorname{MSE}\left(\hat{\bar{Y}}_{P r}\right) \geq 0 \\
\left(\frac{1-f}{n}\right) \bar{Y}^{2}\left(C_{y}{ }^{2}+C_{x}{ }^{2}+2 \mathrm{C}_{\mathrm{xy}}\right)-\bar{Y}^{2}\left(\left(\alpha \lambda_{1}+(1-\alpha) \lambda_{2}-1\right)^{2}+\left(\frac{1-f}{n}\right)\left\{C _ { y } { } ^ { 2 } \left(\alpha \lambda_{1}+\right.\right.\right. \\
\left.(1-\alpha) \lambda_{2}\right)^{2}+\theta^{2} C_{x}{ }^{2}\left(3 \alpha^{2} \lambda_{1}{ }^{2}+(1-\alpha)^{2} \lambda_{2}{ }^{2}-2 \alpha \lambda_{1}\right)+2 \theta \mathrm{C}_{\mathrm{xy}}\left(\alpha \lambda_{1}-(1-\alpha) \lambda_{2}-\right. \\
\left.\left.2\left(\alpha^{2} \lambda_{1}{ }^{2}-(1-\alpha)^{2} \lambda_{2}{ }^{2}\right)\right\}\right) \geq 0 \\
\left(\frac{1-f}{n}\right) C_{y}{ }^{2}\left(1-\left(\alpha \lambda_{1}+(1-\alpha) \lambda_{2}\right)^{2}\right) \geq\left(\left(\alpha \lambda_{1}+(1-\alpha) \lambda_{2}-1\right)^{2}+\right. \\
\left(\frac{1-f}{n}\right)\left\{C_{x}{ }^{2}\left(\theta^{2}\left(3 \alpha^{2} \lambda_{1}{ }^{2}+(1-\alpha)^{2} \lambda_{2}{ }^{2}-2 \alpha \lambda_{1}\right)-1\right)+2 C_{\mathrm{xy}}\left(\theta \left(\alpha \lambda_{1}-(1-\alpha) \lambda_{2}-\right.\right.\right. \\
\left.\left.\left.\left.2\left(\alpha^{2} \lambda_{1}{ }^{2}-(1-\alpha)^{2} \lambda_{2}{ }^{2}\right)\right)-1\right)\right\}\right) \\
\rho \geq \frac{(A-1)^{2}+\left(\frac{1-f}{n}\right)\left\{\left(A^{2}-1\right) C_{y}{ }^{2}+C_{x}{ }^{2}\left(\theta^{2}\left(A^{2}+(A+B)(B-1)\right)-1\right)\right\}}{\left(\frac{1-f}{n}\right) 2 C_{x} C_{y}(\theta B(2 A-1)+1)}
\end{gathered}
$$

Case (5): The proposed estimator is more efficient than modified product estimator if

$$
\begin{gathered}
V\left(\hat{\bar{Y}}_{M P}\right)-M S E\left(\hat{\bar{Y}}_{P r}\right) \geq 0 \\
\left(\frac{1-f}{n}\right) \bar{Y}^{2}\left(C_{y}{ }^{2}+\theta^{2} C_{x}{ }^{2}+2 \theta \mathrm{C}_{\mathrm{xy}}\right)-\bar{Y}^{2}\left(\left(\alpha \lambda_{1}+(1-\alpha) \lambda_{2}-1\right)^{2}+\left(\frac{1-f}{n}\right)\left\{C _ { y } { } ^ { 2 } \left(\alpha \lambda_{1}+\right.\right.\right. \\
\left.(1-\alpha) \lambda_{2}\right)^{2}+\theta^{2} C_{x}{ }^{2}\left(3 \alpha^{2} \lambda_{1}{ }^{2}+(1-\alpha)^{2} \lambda_{2}{ }^{2}-2 \alpha \lambda_{1}\right)+2 \theta \mathrm{C}_{\mathrm{xy}}\left(\alpha \lambda_{1}-(1-\alpha) \lambda_{2}-\right. \\
\left.\left.2\left(\alpha^{2} \lambda_{1}{ }^{2}-(1-\alpha)^{2} \lambda_{2}{ }^{2}\right)\right\}\right) \geq 0 \\
\left(\frac{1-f}{n}\right) C_{y}{ }^{2}\left(1-\left(\alpha \lambda_{1}+(1-\alpha) \lambda_{2}\right)^{2}\right) \geq\left(\left(\alpha \lambda_{1}+(1-\alpha) \lambda_{2}-1\right)^{2}+\right. \\
\left(\frac{1-f}{n}\right)\left\{\theta^{2} C_{x}{ }^{2}\left(3 \alpha^{2} \lambda_{1}{ }^{2}+(1-\alpha)^{2} \lambda_{2}{ }^{2}-2 \alpha \lambda_{1}-1\right)+2 \theta \mathrm{C}_{\mathrm{xy}}\left(\alpha \lambda_{1}-(1-\alpha) \lambda_{2}-2\left(\alpha^{2} \lambda_{1}{ }^{2}-\right.\right.\right. \\
\left.\left.\left.\left.(1-\alpha)^{2} \lambda_{2}{ }^{2}\right)-1\right)\right\}\right) \\
\quad \rho \geq \frac{(A-1)^{2}+\left(\frac{1-f}{n}\right)\left\{\left(A^{2}-1\right) C_{y}{ }^{2}+\theta^{2} C_{x}{ }^{2}\left(\left(A^{2}+(A+B)(B-1)\right)-1\right)\right\}}{\left(\frac{1-f}{n}\right) 2 \theta C_{x} C_{y}(B(2 A-1)+1)} \\
\text { where } A=\alpha \lambda_{1}+(1-\alpha) \lambda_{2} \text { and } B=\alpha \lambda_{1}-(1-\alpha) \lambda_{2}
\end{gathered}
$$


